\title{
Systems \& Methods in Extreme Sports Medicine
}

\author{
F. Feletti ${ }^{1,2}$, A. P. Moorhead ${ }^{3}$, O. Mei-Dan ${ }^{4}$ \\ 1 Department of Diagnostic Imaging, Ausl Romagna, S. Maria delle Croci Hospital, Ravenna, Italy \\ 2 Department of Electronics, Information and Bioengineering, Politecnico di Milano University, Milan, Italy \\ ${ }^{3}$ Department of Mechanical Engineering, Politecnico di Milano University, Lecco, Italy \\ ${ }^{4}$ CU Sports Medicine and Hip Preservation, Department of Orthopedics, University of Colorado School of \\ Medicine, Boulder, Colorado, USA
}

DOI:

10.32098/mltj.02.2020.20

Extreme sports are associated with increased risk due to the inherently dangerous maneuvers performed, the environmental variables, and the distance from medical assistance. The majority of extreme sports disciplines were developed or popularized in the final decades of the previous century and are now practiced by millions of participants around the globe, from recreational adventure sports enthusiasts to elite athletes. Over the previous years the media has created grandiose spectacles of extreme sporting events, choosing to highlight crashes and the inflection points between risk and reward.

The mainstream media continuously reinforces the risk of these sports by reporting major injuries or fatalities. The extensive hours of training and safety protocol practice often takes a back seat during the coverage of extreme sports, creating a representation of inflated risk that seems insurmountable to the general population. Similar to other sports, extreme sports begin with the development of a fundamental set of basic skills before progressing to a riskier or a competitive version of the sport. Upon entry into extreme sports the first lesson is always to protect the environment and the safety of oneself. These principles are continually reinforced as the highest priority throughout every branch of extreme sports. As athletes progress into more challenging environments, both physical and psychological safety considerations follow a direct relationship with the amount of risk at hand.

The field of extreme sports is rapidly expanding with new and extreme versions of existing sports and the introduction of novel extreme disciplines. The rise in popularity and spread of legitimacy accompanied the introduction of extreme sports into the Olympics. The variety of extreme sports on land, water, and in the air, have merged to create multi-sport races that can take place over several days and a large geographical area. Whether it be competition against one another, against oneself, or a challenge with mother nature, extreme sports athletes approach the field with evident tenacity, experience, skill and determination. These unique circumstance are often associated with equally uncommon injuries that require specialized medical attention. Attention that is often common with more traditional sports.

The rapid increase of global participation in outdoor and adventure sports is associated with increased frequency of unique type of injuries. While the proportion of people who advance to a professional level remains low, the injuries affect every level of participation. Future research is further elucidating the predisposing factors and common mechanisms of injury to advance the safety of the sports alongside the advance of the sports themselves. The goal of extreme sports medicine research is to enhance the safety of the sports without compromising their exciting nature. The complexity of these sports provides a framework that is best understood from within the field as first-hand experience. Additionally, participating in the activities provides an avenue to personally connect with athletes and obtain their approval for data collection and analysis. Being personally involved in the sports has fostered our motivation and benefited our research experience.

Our involvement in extreme sports continuously reinforces our drive to expand the medical knowledge surrounding the field. The relatively small amount of published literature provided an opportunity to amass a comprehensive text on extreme and adventure sports injuries. This 
issue combines elements of epidemiology, orthopaedics, engineering, psychology, physiotherapy and many other fields for a multidisciplinary perspective when approaching medical treatment of these injuries. Each chapter is written by a physician, experienced athlete or physiotherapist involved in the sport at an international level. Some chapters present new research while all chapters contain also a review of current literature. The research is presented from an academic viewpoint but is interpreted for the education of all those involved in extreme sports.
The editors aim to present this information for all those interested in treating or partaking in extreme sports. The hope is to bring the literature surrounding the various areas of extreme sports discussed into one volume. Within this review the common patterns and mechanisms of injury, treatment options and analysis of the psychological aspects are presented alongside the similarities and differences of each sport. Hopefully, this will provide useful information to those who enjoy and/or treat the fantastic world of extreme sports, and set a framework for future research to come. 